\title{
Research on Analysis System for Trace hydrogen sulfide content Based on threshold method of Heursure De-noising and TDLAS
}

\author{
Taoyi Xu ${ }^{1, a}$, Cheng Zhong ${ }^{1, b}$ \\ ${ }^{1}$ City College of Wuhan University of Science and Technology, Wuhan 430083, China; \\ a158730244@qq.com, b 363858170@qq.com
}

\begin{abstract}
Keywords: Mallat fast algorithm; TDLAS Technology; Heursure threshold; Hydrogen sulfide; Measurement accuracy; concentration detection.
\end{abstract}

\begin{abstract}
In this paper, an analysis system for trace hydrogen sulfide is given in this paper since traditional hydrogen sulfide pollution monitoring technique has such defects as high costs, cumbersome process and bad real time performance. Samples of natural gas can be taken for analysis by using a TDLAS zero-contact technique. $\mathrm{H}_{2} \mathrm{~S}$ components can be separated by Mallat wavelet algorithm. It reduces signal noise effectively by applying the Heursure threshold method, so that the pollutants will not affect the accuracy of the system measurement. Experimental results show that detection of the maximum error of this analysis system is only 5\%. Compared with other methods, experimental results show that this system has a higher response speed. Its high response speed which reflects in time the change of concentration of hydrogen sulfide helps people to control the production status.
\end{abstract}

\section{Introduction}

Hydrogen sulfide $\left(\mathrm{H}_{2} \mathrm{~S}\right)$ is one of the most common impurities in natural gas. High content of hydrogen sulfide in natural gas will accelerate the corrosion of metal pipes and the aging of non-metallic materials. Gas escaping will lead to serious safety accidents, so it is necessary to monitor and control the concentration of hydrogen sulfide strictly $\left(<20 \mathrm{mg} / \mathrm{Nm}^{3}\right)$.

Before removing hydrogen sulfide from natural gas, it is necessary to detect the content of hydrogen sulfide. At present the main detection methods applied can be divided into two categories: the first one is laboratory determination with field sampling, this method has high measurement accuracy, but the working process is tedious, and the data obtained is seriously lagging behind; the other one is field determining method, this method is favorable for the field test, but the error is big during the test. It is easy to be disturbed by the field environment and the result can be affected easily. More convenient and effective measurement methods are needed to solve these problems.

The gas analysis system described in this paper is based on the TDLAS laser on-line analysis technique and the Mallat fast wavelet analysis algorithm to detect the natural gas containing hydrogen sulfide and process the collected data. The system responds quickly and is not affected by the contamination. This method provides the latest high-tech solution for the quality and safety management of natural gas

\section{System Architecture}

The system consists of three functional modules, which are: laser detecting unit, photoelectric detection acquisition unit and data storage processing unit, as shown in figure 1.

The laser detecting unit comprises a gas absorption pool, a laser emitter, a laser calibration device, a temperature control module, etc. This unit lets the laser accurately enter the long path gas absorption pool, and then makes it pass through a certain concentration of the gas to be measured when testing the concentration of hydrogen sulfide. The photoelectric detecting and collecting unit comprises a laser detecting driver, a preamplifier filter, a phase lock amplifier and a dynamic data acquisition card. This unit first detects the light intensity of the reflected light beam through the absorption cell by the photoelectric detector, then analyzes and preprocesses it, then converts it to a current signal and at 
last sent it to the data processing unit. Data storage and processing are implemented in the upper computer. Data noise is filtered out by using Matlab software based on Mallat fast algorithm. And we can get the results of the data finally.
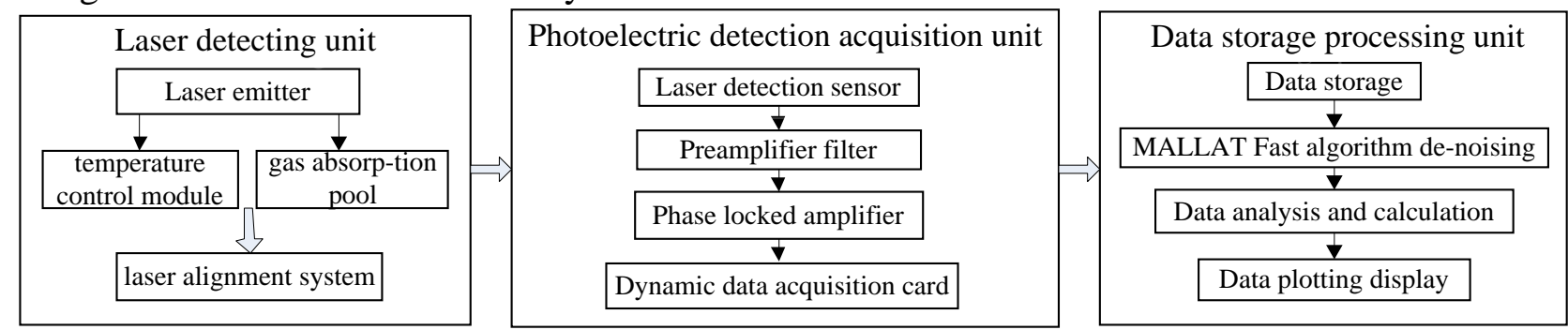

Fig. 1 Schematic of $\mathrm{H}_{2} \mathrm{~S}$ content analysis of system

\section{TDLAS sampling analysis and Mallat wavelet de-noising}

TDLAS sampling analysis. The system employs tunable diode laser absorption spectroscopy (TDLAS) to detect the presence of trace substances in natural gases. Absorption spectroscopy is a widely used technique for sensitive trace species detection. The measurement is more accurate and significantly more reliable than traditional surface-based sensors that are subject to surface contamination.

According to the Beer-Lambert absorption law, $\mathrm{N}$ represents the species concentration. Thus, the ratio of the absorption measured when the laser is tuned on-resonance versus off-resonance is directly proportional to the number of molecules of that particular species in the beam path, is given by

$$
N=\frac{-1}{\sigma(\lambda) l} \ln \left[\frac{I(\lambda)}{I_{0}(\lambda)}\right]
$$

The first step is to launch ramp current signal to the laser transmitter to drive it to emit laser when it works. After the laser beam pass through the gas chamber, the concentration of hydrogen sulfide in gas can be calculated by the formula (1) according to the spectrum signal received by detector.

In order to avoid the interference of the system from the laser transmitter, the background gas and the circuit noise, the system takes the fundamental absorption spectroscopy concept a step further by using a sophisticated signal detection technique called wavelength modulation spectroscopy (WMS). When employing WMS, the laser drive current is modulated with a $\mathrm{kHz}$ sine wave as the laser is rapidly tuned. A lock-in amplifier is then used to detect the harmonic component of the signal that is at twice the modulation frequency (2f). This phase-sensitive detection enables the filtering of low-frequency noise caused by turbulence in the sample gas, temperature and/or pressure fluctuations, low-frequency noise in the laser beam or thermal noise in the detector.

Heursure soft-threshold method. When data sampled by the photoelectric detector is converted to electric signal, the noise signal is often accompanied with data outputting, such as shot noise, thermal noise, which affect the next analysis adversely. So the Mallat algorithm is used in wavelet decomposition and reconstruction of sampling signals. Noise can be effectively removed on the traversal whole frequency range by using the wavelet threshold method. This method can extract data accurately, so as to improve the detection accuracy.

Mallat wavelet analysis technology decomposed the signals which need to divide component into different frequency and time domains according to certain rules. When the signal is decomposed to a certain extent, wavelet multi-resolution decomposes signal in low frequency component, which is considered the fundamental signal. The noise signals are generally concentrated in the high frequency domains. The high frequency components could be processed by wavelet threshold method to achieve de-noising purpose.

Db8 is selected for the wavelet signal decomposition, and decomposition scale is 3 . The three layers wavelet decompositions of original signal $S_{0}$ is expressed as: $S_{0}=S_{3}+d_{3}+d_{2}+d_{1}$. In this formula, $\mathrm{Si}$ and di $(\mathrm{i}=1,2,3)$ represents frequency components of the signal in each decomposition level. 


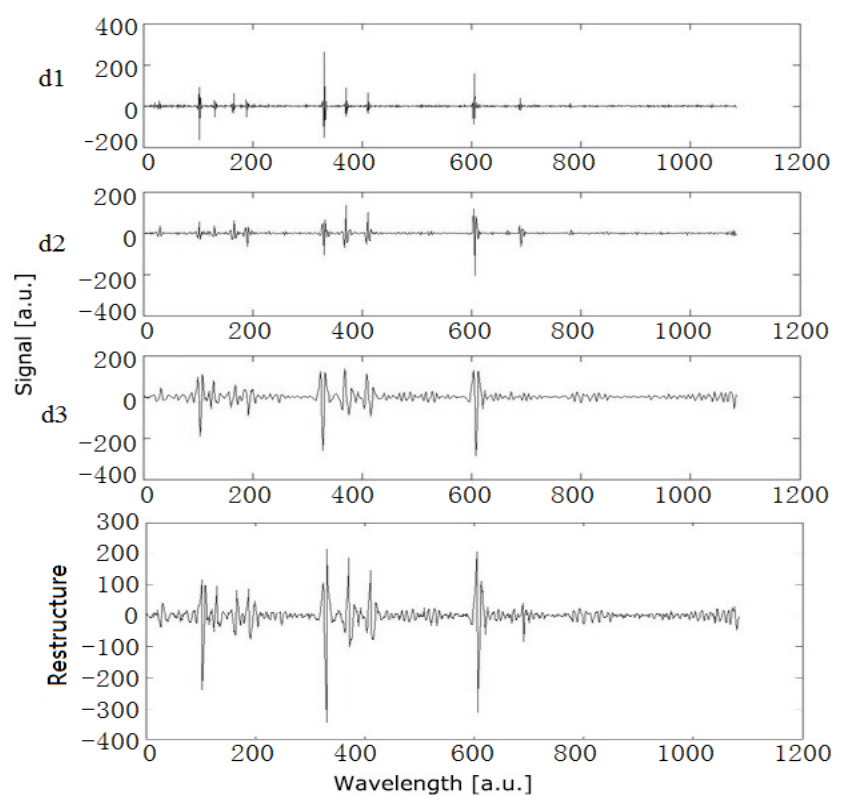

Fig. 2 data decomposition of d3、d2、d1 layers and the reconstructed waveform

Signal coefficient is larger than the noise coefficient through the wavelet decomposition. Wavelet threshold method is used to process the decomposed signal by selecting the appropriate parameters in each decomposed layer (wavelet threshold). There are two commonly used methods: hard threshold and soft threshold method. As the continuity of the reconstructed signal by the hard threshold method is poor. It may cause oscillation which lead to a pseudo Gibbs phenomenon, so the soft threshold method is selected for the system. The wavelet threshold is $\Delta \mathrm{x}$. It is considered to be caused by noise if the coefficient is less than the value, then set it to zero. The remaining value shrink to zero. As shown in a formula (2).

The expression of the soft threshold method is:

$$
y=\left\{\begin{array}{l}
\operatorname{sign}(x) \times(|x|-\Delta x),|x|>\Delta x \\
0, \quad|x| \leq \Delta x
\end{array}\right.
$$

How to select the wavelet threshold value $\Delta \mathrm{x}$ will directly affect de-noising effect of the system according to formula (2). There are many literatures that revealed a variety of methods to solve this problem, such as: SureShrink threshold method, the VisuShrink threshold method, the Heursure threshold method, etc. Heursure threshold Method combines SureShrink together with VisuShrink threshold method. It accentuates the positive and eliminates the negative. The threshold can be determined by using this optimal prediction method according to the SNR, to attain better experimental effect.

$$
\Delta x_{H}=\left\{\begin{array}{l}
\Delta x_{v}, \eta<\mu \\
\min \left(\Delta x_{v}, \Delta x_{s}\right), \quad \eta \geq \mu
\end{array}\right.
$$

$\triangle \mathrm{x}_{\mathrm{V}}$ represents the VisuShrink threshold, and $\triangle \mathrm{x}_{\mathrm{s}}$ represents the SureShrink threshold, and $\eta=\frac{\sum_{k=1}^{N} W_{k}^{2}-N}{N}, \quad \mu=\frac{\left(\log _{2} N\right)^{3 / 2}}{N^{1 / 2}}$.

After the coefficient of the soft threshold processing, the $\mathrm{d} 1 \mathrm{~d} \mathrm{~d} 2$ and $\mathrm{d} 3$ are extracted to reconstruct new data characteristic curve, as shown in figure2. It can remove most of the noise from the original signal effectively. The tuning laser of original data is positive when passing through the slope current tunable laser, which not only increases the wavelength of the current, but also makes the corresponding output power increased. Output fluctuation can be eliminated by adjusting the intensity of the laser output signal. 


\section{Simulation and Data Analysis of the system}

After denoising by Mallat wavelet, the real data collected by the system can be effectively separated from the noise, which has laid a good foundation for further data calculation. Figure 3 shows the typical raw data from a laser absorption spectrometer scan including the incident laser intensity, $I_{0}(\lambda)$, and the transmitted intensity, $I(\lambda)$, for a clean system and one with contaminated mirrors (shown to illustrate the system's relative insensitivity to mirror contamination). Note that contamination of the mirrors results solely in lower overall signal. However, by tuning the laser off-resonance as well as on-resonance and normalizing the data, this technique calibrates itself every scan, resulting in measurements that are unaffected by mirror contamination.

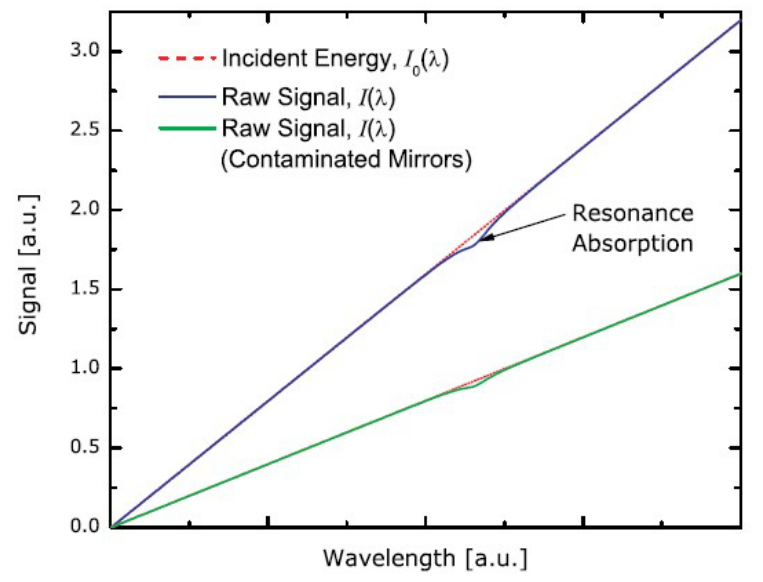

Fig 3 Typical raw signal from a laser diode absorption spectrometer with and without mirror contamination

Based on the existing research, it is known that the center absorption spectrum of the vibration line of hydrogen sulfide gas $\mathrm{v} 1+\mathrm{v} 2+\mathrm{v} 3$ is about $1587.5 \mathrm{~nm}$. Therefore, when measuring the concentration of hydrogen sulfide gas, a diode laser with a wavelength of 1400 to $2000 \mathrm{Nm}$ is selected for the system, so that it can minimize the error rate of the mixed hydrocarbon background gas. And it takes indium gallium arsenic photodiode with the characteristics of high quantum efficiency, low capacitance, low dark current, sensitive measurement as the experimental detector.

In the experiment, let the laser enter the gas chamber and pass through the experimental sample gas, the light intensity signal can be detected by detector after being absorbed by the hydrogen sulfide, and converts it into electrical signals, this current signal is received by the host computer, then we can get $\mathrm{H}_{2} \mathrm{~S}$ concentration value according to the calculated formula by removing noise data. Table 1 is the comparison of the content of hydrogen sulfide in the system test and the actual sample gas.

Table 1 The content of hydrogen sulfide in the system test and the actual sample gas comparing

\begin{tabular}{cccc}
\hline Numble & Actual concentration (PPM) & Test concentration (PPM) & Error rate(\%) \\
\hline 1 & 50 & 47.68 & 4.86 \\
2 & 150 & 143.02 & 4.88 \\
3 & 250 & 240.97 & 3.74 \\
4 & 350 & 337.03 & 3.84 \\
5 & 450 & 430.96 & 4.41 \\
6 & 550 & 526.96 & 4.37 \\
7 & 650 & 620.04 & 4.83 \\
8 & 750 & 717.92 & 4.46 \\
9 & 850 & 811.98 & 4.68 \\
10 & 950 & 907.13 & 4.73 \\
\hline
\end{tabular}

The results show that the low noise signal generated by this method with the later use of fast processing algorithm analysis, the highest real-time response rate (about 1 second) detection sensitivity can reach $10^{-4}$ (PPM), the detection sensitivity depends on the target and the background gas component, but with the increase of $\mathrm{H}_{2} \mathrm{~S}$ concentration, the error becomes larger, however, the maximum error is controlled within $5 \%$, the effect is satisfactory. 
In response rate contrast experiment with the traditional hydrogen sulfide detection methods, a more commonly used hydrogen sulfide alarm equipment is selected for testing. This equipment uses electrochemical sensors to detect the gas concentration in a natural diffusion mode. In this experiment, two devices are used to detect the same gas, and change the content of hydrogen sulfide in the sample gas gradually, then record the actual measurement values of two measuring devices and the corresponding time, plot drawing as shown in figure 4.

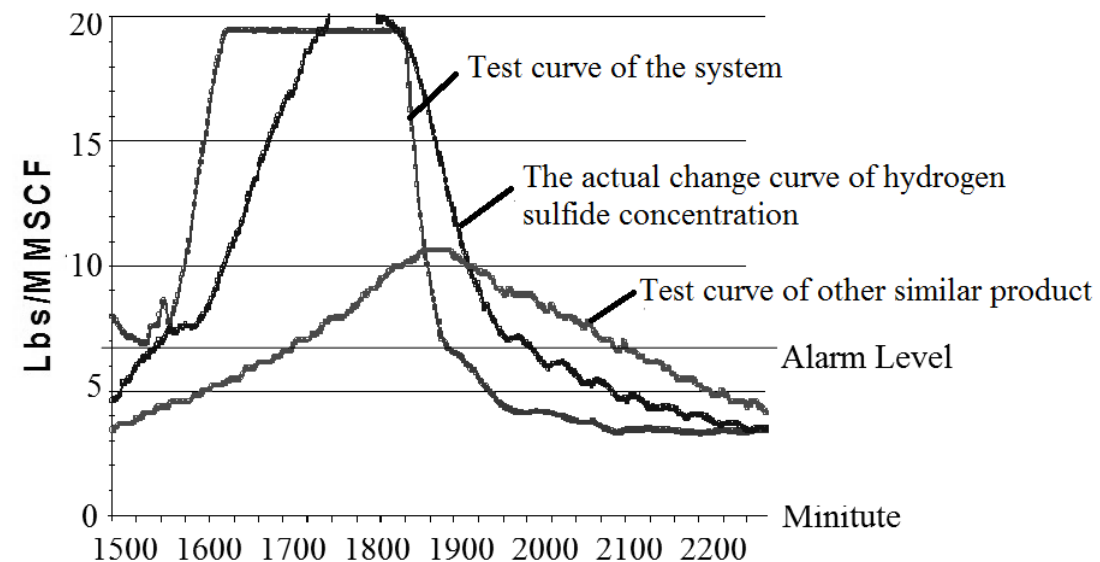

Fig. 4 comparison experiment curve of response rate detection

Compared with the experimental curve, it can be seen that the experimental system has a more rapid response to the change of the concentration of hydrogen sulfide, and the experimental data is closer to the true value than the traditional test equipment.

\section{Summary}

The hydrogen sulfide analysis system uses technology of TDLAS laser analysis on line for gas detection. Mallat fast wavelet analysis algorithm is used to process the collected data in the software design, which can effectively improve the ability of real-time analysis and measurement accuracy of natural gas quality. Practice shows that the control system has strong anti-interference, stable and reliable operation. It can avoid the inconvenience of collecting and inspecting the sample gas, save the cost, and obtain satisfactory test results.

\section{References}

[1] Li Changwu, Wang Yutian, Research on the Detecting of the Sulfur Dioxide Polluted Gas Based on Wavelet Transform. Journal of Shenyang Jianzhu University (Natural Science).2008.24(4): 704707

[2] Wang Xiufang, DUAN Yubo. Simulation Study on the Method of Wavelet De-noising. Techniques of Automation and Applications,2004.23(3):21-23

[3] Tao Shiquan. An Active Core Fiber Optic Sensor for Detecting Trace H2S at High Temperature Using a Cadmium Oxide Doped Porous Silica Optical Fiber as a Transducer [J]. Sensorsand A ctuators, B: Chemical 2007, B127(2):471- 479.

[4] Donoho DL.De -noising by soft -thresholding[J] .IEEE Trans .On IT, 1995.41(3):613-627

[5] Tang Donglin, Wang Ying Optical $\mathrm{H}_{2} \mathrm{~S}$ Gas Sensor Based on Spectrum-Absorpt ion, Chinese journal of sensors and actuators 